\title{
DENSE SUBGROUPS OF COMPACT GROUPS
}

\author{
HOWARD J. WILCOX
}

Abstract. A new short proof is given showing that infinite compact groups contain dense pseudocompact subgroups of small cardinality. Relationships between connectedness and divisibility in pseudocompact groups are given. It is shown that most compact connected Abelian groups contain proper dense connected subgroups.

1. Pseudocompact subgroups of compact groups. Let $G$ be a topological group and $w(G)$ the smallest cardinal of a base of neighborhoods for the topology of $G$. The following theorem generalizes a result of Itzkowitz [5].

THEOREM 1. If $G$ is an infinite compact group with $w(G)=2^{n}$, then $G$ contains a dense pseudocompact subgroup $H$ with cardinal $\leqq n^{\aleph_{0}}$.

A proof of Theorem 1 is contained in [8], but a much shorter and more elegant proof is available using a theorem of Kuz'minov [6].

TheOREM (Kuz'minov). Every compact group $G$ is the continuous image of some product space $\{0,1\}^{m}$ where $\{0,1\}$ is the two element group with the discrete topology.

It is easy to show (see [2]) that $m$ can be taken equal to $w(G)$ so that $G$ is the continuous image of $\{0,1\} w(G)$.

Proof of Theorem 1. Let $w(G)=2^{n}$ for some infinite cardinal $n$. From [5] we know that $\{0,1\} 2^{n}$ regarded as an Abelian group contains a subset $S$ of cardinal $\leqq n^{\aleph_{0}}$ which meets each nonempty Baire set in $\{0,1\} 2^{n}$. The continuous image of $S$ in $G$ has cardinal $\leqq n^{\aleph_{0}}$ and meets each nonempty Baire set of $G$. By a theorem of Comfort and Ross [1], the group generated by "the continuous image of $S$ is the desired dense pseudocompact subgroup $H$ of cardinal $\leqq n^{\aleph_{0}}$. (Note that the cardinal of $G$ is $2^{2^{n}}$.)

2. Connected and divisible pseudocompact subgroups. It is also shown by Comfort and Ross [1] that when $H$ is a dense pseudocompact subgroup of a compact group $G$, then $G$ is the Stone-Cech

Received by the editors July 6, 1970 .

AMS 1970 subject classifications. Primary 22C05; Secondary 54H10.

Key words and phrases. Compact group, dense pseudocompact subgroup, divisible subgroup, dense proper connected subgroup.

Copyright (c) 1971, American Mathematical Society 
compactification of $H$. It is well known (see [3]) that $H$ is connected if and only if $\beta H$, its Stone-Čech compactification, is connected. In light of this it is tempting to conjecture that the following theorem of Mycielski [7] for compact groups also holds for pseudocompact groups.

TheOREM (Mycielski). A compact group $G$ is divisible if and only if it is connected.

Lemma 1. If $H$ is a dense divisible subgroup of a compact group $G$, then $G$ is divisible.

Proof. Given $g \in G$ and an integer $n \neq 0$, there exists a net $h_{i}$ in $H$ which converges to $g$. Since $H$ is divisible, for each $i$ there is an $x_{i} \in H$ such that $x_{i}^{n}=h_{i}$. Now the net $x_{i}$ in the compact set $G$ has a subnet $x_{i_{k}}$ converging to some point $x \in G$. Clearly $x^{n}=g$.

Theorem 2. If a group $H$ is pseudocompact and divisible, then it is connected.

Proof. By Lemma $1, \beta H$ is a compact divisible group and thus is connected by Mycielski's Theorem. It follows that $H$ is connected.

Unfortunately, connected does not imply divisible for pseudocompact groups as the following example shows.

EXAMPLE $1 .^{1}$ Let $F$ be the group of real numbers modulo 1 under addition modulo 1 . Let $G$ be the product $F^{n}$ for some uncountable cardinal $n$. Clearly $G$ is connected and divisible. Let $H=\left\{x \in G: x_{i}=0\right.$ for all but at most countably many $i\}$. Then $H$ is a dense pseudocompact subgroup of $G$ (see [1]). The group $K$ generated by $H$ and the point $p$ all of whose coordinates are $\sqrt{ } 2 / 2$ is pseudocompact and connected but is not divisible because $p \neq 2 x$ for any $x \in K$.

Thus connected pseudocompact groups are not necessarily divisible and dense subgroups of compact divisible groups are not necessarily divisible.

3. Connected subgroups of compact groups. A topological group is said to be solenoidal if it contains a dense continuous homomorphic image of the real numbers.

LEMMA 2. If $G$ is a compact group which is not topologically isomorphic with the circle group $T$ and if $G$ is solenoidal, then $G$ contains a proper dense continuous homomorphic image of the reals.

PRoof. If $G$ is solenoidal but contains no proper dense continuous homomorphic image of the reals, then there exists a continuous

\footnotetext{
${ }^{1}$ The author wishes to thank Professor W. W. Comfort for his suggestions.
} 
homomorphic mapping of the reals onto $G$. By 5.27 and 5.29 of [4] this mapping is open and $G$ is topologically isomorphic with the reals modulo the kernel of the mapping. Thus $G$ is topologically isomorphic with $T$.

It is known (see [4]) that a compact Abelian group $G$ is solenoidal if and only if $G$ is connected and $w(G) \leqq c$.

ThEOREM 3. Every compact connected Abelian group except $T$ contains a dense proper connected subgroup provided that $w(G) \leqq c$ or $w(G)^{\aleph_{0}}=w(G)$.

Proof. If $w(G) \leqq c$, then $G$ is solenoidal and the theorem follows from Lemma 2. If $w(G)^{\aleph_{0}}=w(G)$, then the theorem follows from Theorem 1 and the fact that a dense pseudocompact subgroup of $G$ is connected if and only if $G$ is connected. In this case the dense proper connected subgroup will be pseudocompact.

\section{REFERENCES}

1. W. W. Comfort and Kenneth A. Ross, Pseudocompactness and uniform continuity in topological groups, Pacific J. Math. 16 (1966), 483-496. MR 34 \#7699.

2. R. Engelking and A. Pełczynski, Remarks on dyadic spaces, Colloq. Math. 11 (1963), 55-63. MR 28 \#4504.

3. Leonard Gillman and Meyer Jerison, Rings of continuous functions, University Series in Higher Math., Van Nostrand, Princeton, N. J., 1960. MR 22 \#6994.

4. E. Hewitt and K. A. Ross, Abstract harmonic analysis. Vol. I: Structure of topological groups. Integration theory, group representations, Die Grundlehren der math. Wissenschaften, Band 115, Academic Press, New York; Springer-Verlag, Berlin, 1963. MR 28 \#158.

5. Gerald L. Itzkowitz, Extensions of Haar measure for compact connected Abelian groups, Bull. Amer. Math. Soc. 71 (1965), 152-156. MR 30 \#188.

6. V. Kuz'minov, Aleksandrov's hypothesis in the theory of topological groups, Dokl. Akad. Nauk SSSR 125 (1959), 727-729. MR 21 \#3506; errata, MR 21 p. 1599.

7. Jan Mycielski, Some properties of connected compact groups, Colloq. Math. 5 (1958), 162-166. MR 20 \#6479.

8. Howard J. Wilcox, Pseudocompact groups, Pacific J. Math. 19 (1966), 365-379. MR 34 \#5964.

Wellesley College, Wellesley, Massachusetts 02181 